\title{
PARTIAL ALBINISM: A CHALLENGE TO IDENTIFICIATION
}

\author{
ASTRID MACLEOD, 308 Harvard Avenue West, Winnipeg, Manitoba. R2C 1Y6
}

Albinism is an interesting phenomenon that can provide unique challenges to a wildlife rehabilitator. As an orphan care volunteer with the Manitoba Wildlife Rehabilitation Organization (MWRO), I was presented with just such a challenge in May 1993.

A nest of hatchling birds had been removed from a private plane coming out of storage in St. Andrews and delivered to MWRO in Winnipeg. The nest was deep and round, set inside a box of stones. Inside the nest were four, tiny hatchlings, an unmarked blue egg, and a long black and white feather. The hatchlings were quite chilled, obviously having missed more than a few feedings.

After placing the birds in an incubator nest for stabilization, the immediate task was to identify them. The hatchlings were covered with white and pale grey neoptiles, their gapes were a pale pink as were their skin and feet, and their swollen gape flanges were creamy white. Typically this amount of information linked with the shape, colour and size of prominent physical features (e.g., beak, feet, etc.) leads to fast identification. In this particular case, however, such was not to be. I consulted many bird identification books, but every time I thought I had the answer, some small detail was incorrect. The gape flange resembled that of a European Starling, but the gape colour did not correspond. I consulted with other wildlife rehabilitators and biologists but a positive identification could not be made.

During the time that followed, the hatchlings failed to thrive. Perhaps they had been too cold and too long without food, but one by one I watched them fail, until only a single

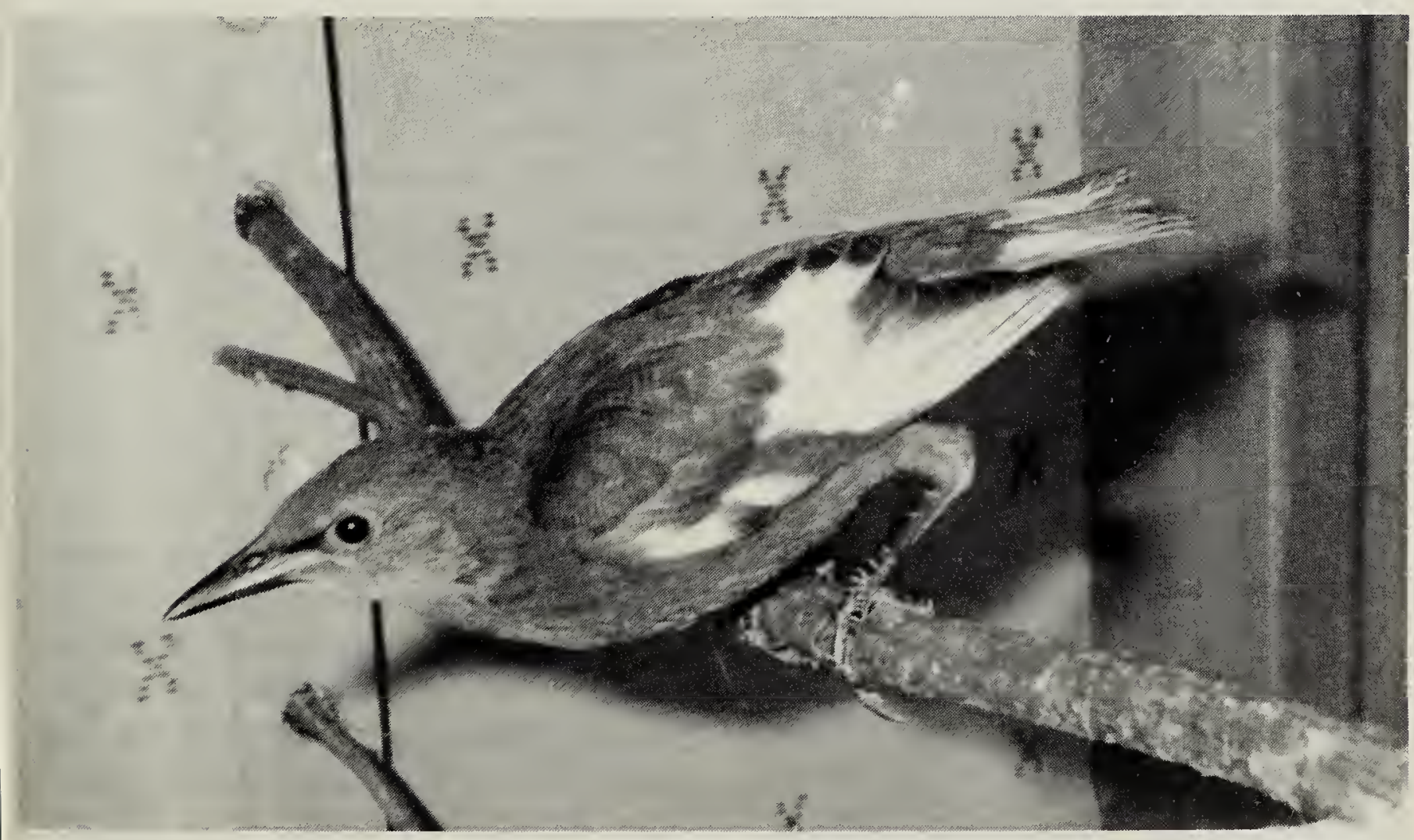

The mystery bird develops markings.

Astrid MacLeod 


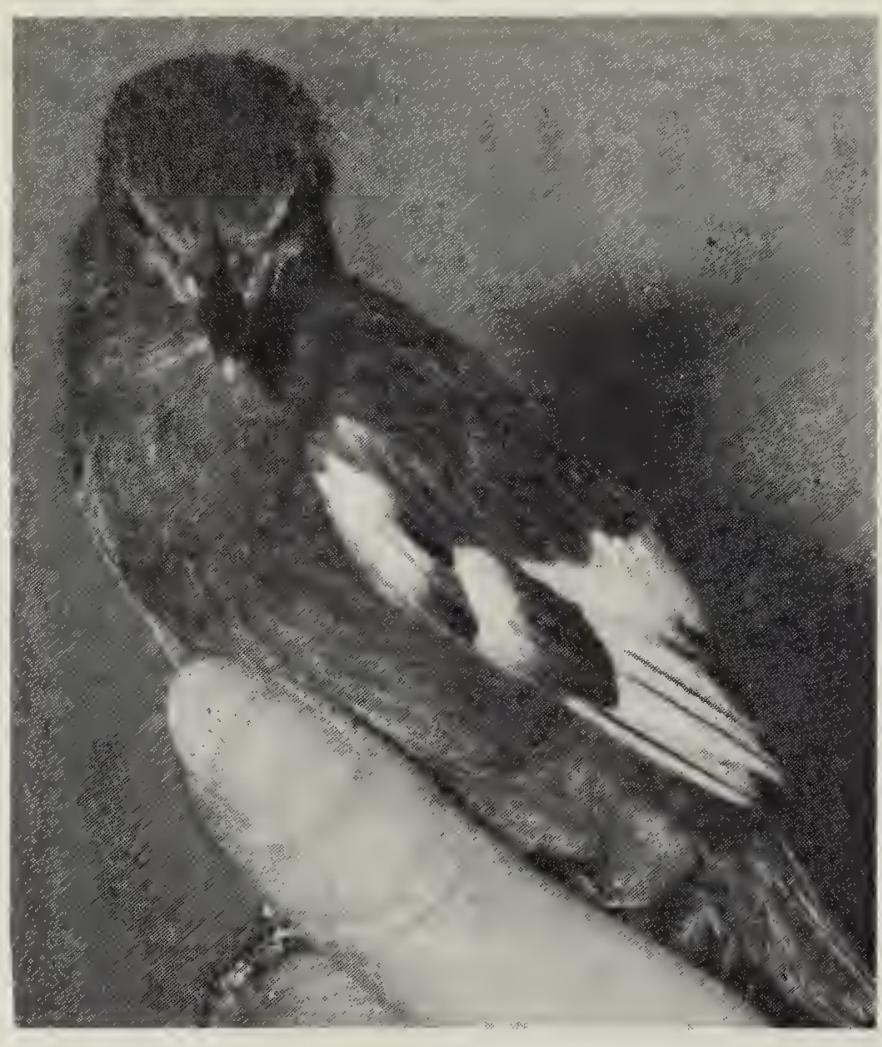

A partial albino European

Starling.

Astrid MacLeod

bird was left. With each passing day, it became increasingly more important to make an accurate identification so that the bird's diet could be made to correspond to its natural needs.

By the time the bird's eyes had opened and it developed pin feathers, a large number of people were attempting to provide an identification. In addition to the bird's physical features, the blue egg and black and white feather that were found in the original nest were added as clues. The egg presented many possibilities, while the feather narrowed the range to a handful of choices, none of which correlated to blue eggs or pink gapes.

As the days passed, the bird's skin darkened to a grey colour and became scaly. The beak elongated and blackened, as did the legs and feet. The feathers began to emerge and were dark charcoal in colour, almost black. Finally, the primary and secondary feathers unfurled ... they were black with white stripes. The tail was yet to appear.

With the lack of identification, feed- ing became quite a concern and worry. To cover all bases, soft-bill diet, monkey chow, seeds, nuts, fruits, insects and even fish were offered during the hand-feeding stage. The bird readily accepted everything and anything. Possibly this omnivorous behaviour signified it to be a magpie or bobolink. Unfortunately, the bird rapidly outgrew bobolink status, and as its tail started to emerge it became obvious that it was not a magpie either.

Now a fully developed fledgling, our mystery bird was deemed physically healthy, but still lacked an identity. Release could not be considered until we ascertained if it could socialize with its own kind.

As luck would have it, the bird maturity finally elicited vocalizations the magic clue. At this point in time, MWRO Centre Manager, Brian Ratcliff, came to look at the bird and upon hearing the bird's song identified it as a European Starling. Subsequent examination revealed that we indeed did have a European Starling, but one with partial albinism of the primaries and secondaries, as well as a band at the end of the tail feathers.

The mystery was solved. The bird was placed with other European Starlings and after three weeks of successful socialization and acclimatization was ready for release. As a good-bye gesture, the bird's breast plumage broke out in the trade-mark speckled pattern just prior to release - confirmation that we had indeed made a correct identification.

As a wildlife rehabilitator, this was my first experience working with partial albinism and I found it to be unbelievably fascinating. The extreme amount of variability in the physical characteristics of young partial albinos presents a unique challenge in providing immediate and proper care. 